\title{
EXTRAÇÃO EM FASE SÓLIDA DE RESÍDUOS DE AGROTÓXICOS DE ÁGUA SUPERFICIAL, EMPREGANDO UM SORVENTE DE MENOR RETENÇÃO
}

\author{
Carlos A. S. Aguiar Júniora, Nadja T. M. Silva ${ }^{\mathrm{a}}$, Dayane F. Soares ${ }^{\mathrm{b}}$ e Anizio M. Faria ${ }^{\mathrm{a}, *}$
}

${ }^{a}$ Faculdade de Ciências Integradas do Pontal, Universidade Federal de Uberlândia, 38304-402 Ituiutaba - MG, Brasil

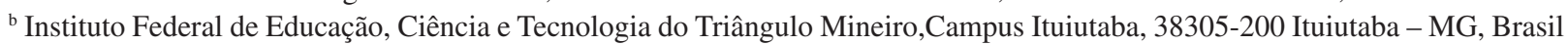

Recebido em 12/02/2018; aceito em 15/03/2018; publicado na web em 29/03/2018

\begin{abstract}
SOLID-PHASE EXTRACTION OF PESTICIDE RESIDUES FROM SURFACE WATER USING A LAB-MADE LOWERRETENTION SORBENT. Solid phase extraction (SPE) is one of the main sample preparation techniques. However, the cost of cartridges and the strong retention of hydrophobic compounds in traditional solid phases are limiting factors for their use. This paper describes the application of lab-made SPE cartridges with low retention material as solid phase, Si(PDAS) - poly(dimethylco-alkylmethylsiloxane) thermally immobilized on silica, in the determination of pesticide residues in surface water samples. The extraction method of three pesticides: carbendazim, diurom and methyl parathion in water samples was developed, optimized and validated using $\mathrm{Si}$ (PDAS) cartridges and HPLC-UV for quantification. The optimized method required a small organic solvent volume for the elution of pesticides from $\mathrm{Si}$ (PDAS), producing a concentration factor of 1000 times and allowing limits of detection like those obtained with hyphenated techniques. The validated method for extraction of pesticides in water samples presented: accuracy, with pesticide recoveries between 70-110\%; precision, with RSD $<20 \%$, and; high detectability, resulting in LOQ $<0.4 \mu g \mathrm{~L}^{-1}$ for all compounds. $\mathrm{Si}(\mathrm{PDAS})$ cartridges showed similar efficiency to those commercial $\mathrm{C}_{18}$ for the extraction of pesticides in surface water samples, with additional advantages such as ease, simplicity and low cost of preparation, up to six times cheaper than commercial cartridges.
\end{abstract}

Keywords: solid phase extraction; low retention sorbent; surface water, pesticides, HPLC-UV, method validation.

\section{INTRODUÇÃO}

A extração em fase sólida (SPE, solid phase extraction) se tornou um dos principais métodos de extração de solutos de amostras líquidas nas últimas décadas..$^{1-6}$ Dentre os principais atrativos da SPE podem ser destacados o alto fator de concentração dos solutos, a possibilidade de realização de inúmeras extrações simultâneas e a redução do uso de solventes orgânicos comparada a técnicas de extração com solventes. ${ }^{7,8}$ Amostras ambientais que apresentam concentrações de contaminantes em níveis de subtraços estão entre as principais aplicações da SPE. ${ }^{9-13}$ Mesmo com a existência de técnicas hifenadas altamente sofisticadas, os níveis de concentração de contaminantes orgânicos em amostras de águas superficiais ou subterrâneas normalmente se encontram abaixo dos limites de detecção destas técnicas, tornando-se necessária a pré-concentração dos compostos, além de seus isolamentos da matriz da amostra antes da medida analítica. ${ }^{7}$

O principal sorvente empregado como fase sólida na SPE é o octadecilsilano $\left(\mathrm{C}_{18}\right)$ ligado quimicamente sobre sílica. ${ }^{14}$ Apesar do registro de uso de outros sorventes para a remoção de contaminantes orgânicos de amostras de água, ${ }^{3,7,8,13} \mathrm{o} \mathrm{C}_{18}$ continua sendo o cartucho mais amplamente empregado para esta finalidade. Essa utilização se deve à seletividade adequada para os contaminantes orgânicos, eficiência no processo de remoção de analitos de amostras de água e a disponibilidade comercial de cartuchos $\mathrm{C}_{18}{ }^{8,14}$ No entanto, fases sólidas a base de $\mathrm{C}_{18}$ quimicamente ligado sobre sílica apresentam alguns inconvenientes, como, por exemplo, a necessidade de solventes mais hidrofóbicos ou de volumes maiores de solventes orgânicos comuns para a remoção de solutos de maior hidrofobicidade da fase sólida; a limitação de uso do cartucho em condições de $\mathrm{pH}>8$, uma vez que este sorvente apresenta recobrimento incompleto da superfície da sílica, solúvel em meio alcalino. ${ }^{8,12,15}$ Além disso, o custo dos

*e-mail: anizio@ufu.br cartuchos ainda é relativamente elevado, principalmente devido às condições de preparação do sorvente, empregando reagentes organossilanos caros. ${ }^{15}$ Desta forma, a preparação de sorventes que possuam seletividade e eficiência similar ao $\mathrm{C}_{18}$, porém com menor retenção para compostos hidrofóbicos e possibilidade de uso em uma faixa de pH mais ampla é uma necessidade para ampliar as aplicações de SPE.

Em um trabalho anterior, ${ }^{16}$ foi apresentado um sorvente a base de sílica recoberta com o polímero poli(dimetil-co-alquilmetilsiloxano) (PDAS), contendo grupos laterais $\mathrm{C}_{16} / \mathrm{C}_{18}$ intercalados com $\mathrm{C}_{1}$, que possui seletividade similar ao sorvente $\mathrm{C}_{18}$, porém com menor hidrofobicidade, requerendo volumes menores de solventes orgânicos na etapa de eluição. O sorvente $\mathrm{Si}$ (PDAS) possui uma camada polimérica de PDAS imobilizada fisicamente sobre as partículas de $5 \mu \mathrm{m}$ de sílica, que promove um melhor recobrimento da superfície do suporte e, consequentemente, menor exposição da superfície da sílica ao contato com a amostra e eluentes, aumentando a vida útil do cartucho mesmo em condições de $\mathrm{pH}>8$. Adicionalmente, os cartuchos de Si(PDAS) empregam um método simples com aparatos, vidrarias e reagentes de baixo custo, tornando-os até seis vezes mais baratos que os cartuchos comerciais tradicionais de $\mathrm{C}_{18}$.

Neste trabalho, os cartuchos recheados com o Si(PDAS) imobilizado termicamente sobre sílica foram avaliados na extração de três agrotóxicos de amostras de água superficiais, coletadas em regiões de cultivo de cana-de-açúcar. Os agrotóxicos foram escolhidos após levantamento dos principais princípios ativos aplicados na região do Triângulo Mineiro para o combate de pragas/doenças e ervas daninhas nas lavouras de cana-de-açúcar. Foi empregada a cromatografia líquida de alta eficiência com detecção ultravioleta para a quantificação do teor de resíduos dos agrotóxicos nas amostras de água. O método de extração em fase sólida com o sorvente Si(PDAS) dos agrotóxicos de amostras de água foi otimizado e validado, de acordo com o protocolo recomendado pela Comunidade Europeia, documento SANTE/11945/2015. ${ }^{17}$ 


\section{PARTE EXPERIMENTAL}

\section{Materiais}

A preparação da fase sólida foi realizada com sílica gel com diâmetro de partículas de 40 a $63 \mu \mathrm{m}$ e de poros de $60 \AA$ (Sigma-Aldrich, Saint Louis, EUA) e poli(dimetilsiloxano- $c o$-alquilmetilsiloxano) (PDAS) (Sigma-Aldrich). Tolueno, clorofórmio, hexano e acetato de etila, todos grau analítico, foram adquiridos da VETEC (Rio de Janeiro, Brasil) ou da Qhemis (São Paulo, Brasil). As fases móveis e soluções padrões foram preparadas com metanol ou acetonitrila, ambos graus cromatográfico e espectroscópico, que foram adquiridos da JT Baker (Phillipsburg, NJ, EUA). A água ultrapura foi obtida de um ultrapurificador da Megapurity (Billerica, MA, EUA), com resistividade de $18,3 \mathrm{M} \Omega \mathrm{cm}$. Todos os solventes foram previamente filtrados, individualmente, em membranas de PTFE de 0,22 $\mu$ m. Sais $\mathrm{NaCl}, \mathrm{NaNO}_{3}$ e $\mathrm{Na}_{2} \mathrm{SO}_{4}$ foram adquiridos da Synth (São Paulo, Brasil). Os compostos estudados: diurom, parationa metílica e carbendazim foram todos padrões analíticos com grau resíduo de pesticidas (Pestanal), com pureza mínima de 99\%, sendo adquiridos da Sigma-Aldrich. Todas as soluções dos agrotóxicos foram preparadas inicialmente na concentração de $1000 \mu \mathrm{g} \mathrm{mL}^{-1}$ (soluções padrão estoque), individualmente e na forma de misturas. As demais soluções foram preparadas a partir das soluções estoque.

\section{Preparação dos cartuchos de extração em fase sólida}

Todos os cartuchos empregados neste trabalho foram preparados em laboratório, seguindo o procedimento desenvolvido por Novais et al. ${ }^{16}$ Resumidamente, porções de sílica pré-ativada a $140{ }^{\circ} \mathrm{C}$ por $12 \mathrm{~h}$ foram adicionadas a uma solução de PDAS a $30 \%(\mathrm{~m} / \mathrm{v})$ em tolueno na proporção de 1:1:4 (m/m/v), sob agitação branda por $3 \mathrm{~h}$ à temperatura ambiente $\left(\sim 28^{\circ} \mathrm{C}\right)$ em uma capela. Em seguida, a suspensão do sorvente foi deixada sob repouso em capela para a evaporação completa do solvente à temperatura ambiente e sem agitação. O material sorvido e seco foi submetido a uma etapa de imobilização térmica em estufa a $110{ }^{\circ} \mathrm{C}$ por $12 \mathrm{~h}$ para fixação do polímero no suporte de sílica. $500 \mathrm{mg}$ da fase sólida Si(PDAS) foram colocados na forma de suspensão (10\% $\mathrm{m} / \mathrm{v}$ em metanol) em seringas de polipropileno de $3 \mathrm{~mL}$. O sorvente foi compactado nas seringas através de centrifugação a $2500 \mathrm{rpm}$ por $5 \mathrm{~min}$, sendo retido por filtros de politetrafluoretileno (PTFE).

\section{Otimização da extração em fase sólida de agrotóxicos de água}

Para desenvolvimento e otimização de um método de extração em fase sólida dos resíduos de agrotóxicos em água, amostras de 50 $\mathrm{mL}$ de água ultrapura foram fortificadas com uma solução padrão dos agrotóxicos, obtendo uma concentração final de $0,5 \mu \mathrm{g} \mathrm{mL} \mathrm{me}^{-1} \mathrm{de}$ cada composto. Essas amostras foram eluídas em cartuchos $\mathrm{Si}$ (PDAS) conectados a um sistema de vácuo Manifold SPE Restek, modelo ResPrep-12 (Bellafonte, PA, EUA). O procedimento de extração consistiu na eluição de $20 \mathrm{~mL}$ de água ultrapura para ativação da fase sólida, seguida da eluição de $50 \mathrm{~mL}$ de amostras de água fortificadas com $0,5 \mu \mathrm{g} \mathrm{mL}^{-1}$ de cada agrotóxico. Após a passagem da amostra, os cartuchos permaneceram por 5 min sob vácuo para secagem da fase sólida. Em seguida, $5 \mathrm{~mL}$ de solvente de menor afinidade (hexano) pelos compostos foram eluídos pelo cartucho para remoção de potenciais interferentes. Por fim, um volume de $0,5 \mathrm{~mL}$ do solvente de eluição (metanol) foi passado pelos cartuchos para recuperação dos resíduos de agrotóxicos.

Para otimização do procedimento de extração pelos cartuchos preparados em laboratório, foram avaliados os solventes de limpeza e de eluição: metanol, acetonitrila, acetato de etila e hexano; o volume de amostras de água: 50 a $1000 \mathrm{~mL}$; o pH da amostra água; a adição de eletrólito: $\mathrm{NaNO}_{3}, \mathrm{NaCl}$ e $\mathrm{Na}_{2} \mathrm{SO}_{4}$, e a concentração do eletrólito: 50 a $500 \mathrm{mmol} \mathrm{L}^{-1}$.

\section{Validação do método}

O método de determinação de resíduos de parationa metílica, diurom e carbendazim em água, empregando a extração em fase sólida com cartuchos $\mathrm{Si}$ (PDAS) preparados em laboratório, foi validado de acordo com o protocolo preconizado pela Comunidade Europeia, documento SANTE/11945/2015. ${ }^{17}$ Os seguintes parâmetros analíticos foram avaliados no estudo de validação: seletividade, linearidade, efeito de matriz, limites de detecção e de quantificação; precisão (repetibilidade e reprodutibilidade dentro do laboratório), exatidão e robustez.

\section{Análise de amostras de águas superficiais coletadas no Triângulo Mineiro, MG-Brasil}

A aplicabilidade dos cartuchos Si(PDAS) preparados em laboratório foi avaliada, de acordo com o método validado, na extração de resíduos de agrotóxicos em três amostras de águas coletadas em ribeirões localizados dentro de regiões de cultivo de cana-de-açúcar no Triângulo Mineiro, MG-Brasil. As amostras foram coletadas de acordo com as coordenadas geográficas apresentadas na Tabela 1.

\section{Análise dos extratos por CLAE-UV}

Todos os extratos obtidos no processo de extração dos resíduos de agrotóxicos das amostras de água foram analisados por cromatografia líquida de alta eficiência com detecção ultravioleta (CLAE-UV), empregando um cromatógrafo a líquido com sistema binário de bombas, modelo ProStar 210 da Varian (Palo Alto, CA, EUA), detector UV ProStar 325 e injetor manual Reodhyne 7725i, com válvula de injeção de $5 \mu \mathrm{L}$. As separações ocorreram em uma coluna MicroSorb $\mathrm{C}_{18}$ (Varian), $5 \mu \mathrm{m}$, com $150 \mathrm{~mm} \times 4,0 \mathrm{~mm}$ d.i. Foi empregado um gradiente de fase móvel MeOH: $\mathrm{H}_{2} \mathrm{O}$, em que a composição variou de 60:40 (v/v) de 0-3 min; 90:10 (v/v) de 3-8 min; 60:40 (v/v) de 8-10 min. A vazão

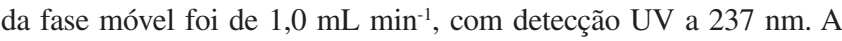
aquisição de dados foi realizada pelo software Galaxie ${ }^{\circledR}$ workstation.

\section{RESULTADOS E DISCUSSÃO}

\section{Otimização do método de extração}

Algumas condições do processo de extração em fase sólida

Tabela 1. Localização dos pontos de coleta das amostras de água

\begin{tabular}{lcccc}
\hline \multirow{2}{*}{ Amostras } & & Coordenadas geográficas & Identificação das amostras \\
\cline { 2 - 4 } & Latitude & Longitude & Elevação $(\mathrm{m})$ & Amostra A \\
\hline Córrego Fundo & $-18,897116$ & $-49,263303$ & 579 & Amostra B \\
Ribeirão dos Baús & $-18,815866$ & $-49,498381$ & 581 & Amostra C \\
Ribeirão Pirapitinga & $-18,689815$ & $-49,371482$ & 455 & Amb \\
\hline
\end{tabular}


empregando os cartuchos $\mathrm{Si}(\mathrm{PDAS})$ foram avaliadas e otimizadas para aplicação na recuperação de resíduos de agrotóxicos de amostras de água. $\mathrm{O}$ pH da amostra, a adição e concentração de eletrólito, o tipo de solvente de limpeza e eluição foram as condições otimizadas na extração.

\section{Escolha dos solventes de eluição e de limpeza}

Os solventes metanol, acetonitrila, acetato de etila e hexano foram avaliados na eluição dos resíduos de agrotóxicos retidos na fase $\mathrm{Si}$ (PDAS). A escolha de solventes de diferentes polaridades se deve à definição do melhor solvente de limpeza e o melhor solvente de eluição no processo de extração em fase sólida. A análise visual dos cromatogramas indicou uma melhor recuperação dos resíduos de agrotóxicos empregando o metanol, por possuir índice de polaridade mais próximo dos compostos, sendo o hexano o pior solvente de eluição por não recuperar os analitos da fase sólida e, portanto, sendo selecionado como solvente de limpeza no processo de extração.

\section{Efeito da adição de eletrólito}

Como os estudos iniciais da extração dos resíduos de agrotóxicos para a escolha do solvente de eluição resultou em baixas recuperações para os agrotóxicos, foi avaliada a possibilidade de aumento da eficiência da extração pela adição de eletrólito às amostras de águas. Esta condição foi avaliada uma vez que os coeficientes de partição octanol-água $\left(\log \mathrm{K}_{\mathrm{ow}}\right)$ são relativamente baixos: 1,52 para o carbendazim, 2,68 para o diurom e 2,86 para a parationa metílica. ${ }^{18}$ Assim, os compostos possuem afinidade maior pela água, sendo mais difíceis de serem retidos na fase $\mathrm{Si}$ (PDAS). A Figura 1 apresenta as recuperações dos agrotóxicos alcançadas na extração com $\mathrm{Si}(\mathrm{PDAS})$ quando à água foram adicionados três diferentes tipos de sais $(\mathrm{NaCl}$, $\mathrm{NaNO}_{3}$ e $\mathrm{Na}_{2} \mathrm{SO}_{4}$ ).

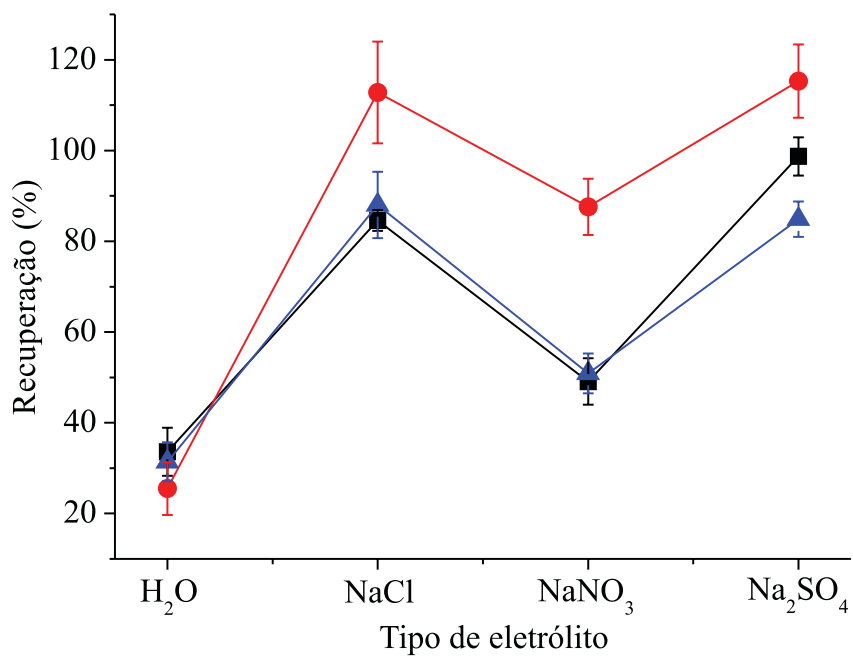

Figura 1. Efeito da adição de eletrólitos na recuperação dos agrotóxicos de água empregando a extração em fase sólida com $\operatorname{Si}(P D A S) . n=3$. $\square$ Carbendazim, $\bigcirc$ diurom e $\boldsymbol{\Delta}$ parationa metílica

A adição de sais na amostra de água resulta em uma melhora na recuperação dos agrotóxicos empregando a fase $\mathrm{Si}(\mathrm{PDAS})$. Esta melhora na recuperação é mais acentuada para a adição de $\mathrm{Na}_{2} \mathrm{SO}_{4}$, de acordo com a Figura 1. O aumento na recuperação dos agrotóxicos da água pode estar associado ao efeito salting out, no qual os sais atraem as moléculas de água do meio, de modo a ficar menos água disponível para a solvatação das moléculas dos agrotóxicos o que acarreta na diminuição da sua solubilidade em água, facilitando a retenção dos agrotóxicos na fase $\mathrm{Si}(\mathrm{PDAS})$ e sua posterior eluição com o metanol. As recuperações aumentaram com a adição de eletrólitos na seguinte ordem: $\mathrm{NaNO}_{3}<\mathrm{NaCl}<\mathrm{Na}_{2} \mathrm{SO}_{4}$. Considerando que o sódio é comum em todos os sais avaliados, os ânions são os responsáveis pelo aumento na recuperação dos agrotóxicos, que pode ainda ser correlacionado com o aumento do raio de hidratação dos íons 165,181 e 244 pm para nitrato, cloreto e sulfato, respectivamente. ${ }^{19}$

A influência da concentração de $\mathrm{Na}_{2} \mathrm{SO}_{4}$ adicionada na amostra de água também foi avaliada na recuperação dos resíduos de agrotóxicos. A faixa de concentração de 50 a $500 \mathrm{mmol} \mathrm{L}^{-1}$ foi estudada. A Figura 2 indica que o aumento da concentração de eletrólito na amostra aquosa leva a uma diminuição da recuperação dos agrotóxicos, que pode estar relacionado ao aumento da solubilidade dos agrotóxicos em água devido à maior concentração dos íons do $\mathrm{Na}_{2} \mathrm{SO}_{4}$ interagirem com as cargas iônicas dos agrotóxicos. Desta forma, a concentração de $50 \mathrm{mmol} \mathrm{L}^{-1}$ de $\mathrm{Na}_{2} \mathrm{SO}_{4}$ na amostra de água foi considerada a melhor condição de extração dos resíduos de agrotóxicos. Torna-se igualmente importante avaliar o efeito do $\mathrm{pH}$ da amostra aquosa no processo de extração com o Si(PDAS), uma vez que as moléculas na forma ionizada não são retidas adequadamente na fase sólida.

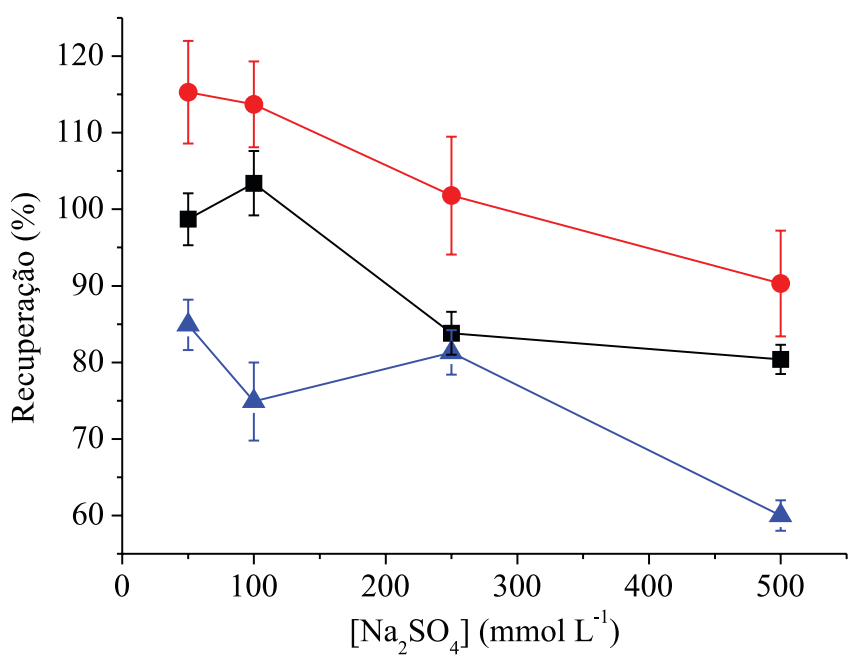

Figura 2. Efeito da concentração de $\mathrm{Na}_{2} \mathrm{SO}_{4}$ na recuperação dos agrotóxicos de água empregando a extração em fase sólida com $\operatorname{Si}(P D A S) . n=3$. - Carbendazim, $\boldsymbol{\bullet}$ diurom e $\boldsymbol{\Delta}$ parationa metílica

Efeito do pH na extração

A escolha do $\mathrm{pH}$ da amostra é um fator importante na extração em fase sólida de multirresíduos de agrotóxicos de água, uma vez que os compostos possuem valores de $\mathrm{pKa}$ variados podendo estarem ionizados de formas distintas na amostra e, assim, apresentarem menor retenção na fase sólida. O estudo de recuperação dos agrotóxicos da fase $\mathrm{Si}(\mathrm{PDAS})$ foi realizado na faixa de $\mathrm{pH} 2$ a 8 das amostras de água, estabelecida como a faixa de $\mathrm{pH}$ estável para materiais à base de sílica. ${ }^{15}$ As recuperações dos compostos em diferentes condições de $\mathrm{pH}$ das amostras de água estão apresentadas na Figura 3.

As maiores recuperações dos agrotóxicos ocorreram quando o $\mathrm{pH}$ das amostras de água esteve próximo da neutralidade, em pH 6 (Figura 3). Nesta condição ocorre a maior retenção dos compostos na fase $\mathrm{Si}$ (PDAS), removendo os agrotóxicos da água com maior facilidade devido à menor quantidade de espécies dos agrotóxicos que se encontram na forma ionizada nessa condição de $\mathrm{pH}$.

\section{Volume de breakthrough}

Uma das principais vantagens do uso da extração em fase sólida na preparação de amostras de compartimento ambiental é sua capacidade de concentrar o analito no extrato final. O fator de concentração será maior quanto maior for o volume de amostra a ser extraído e menor o volume de solvente na etapa de eluição. No entanto, volumes muito 


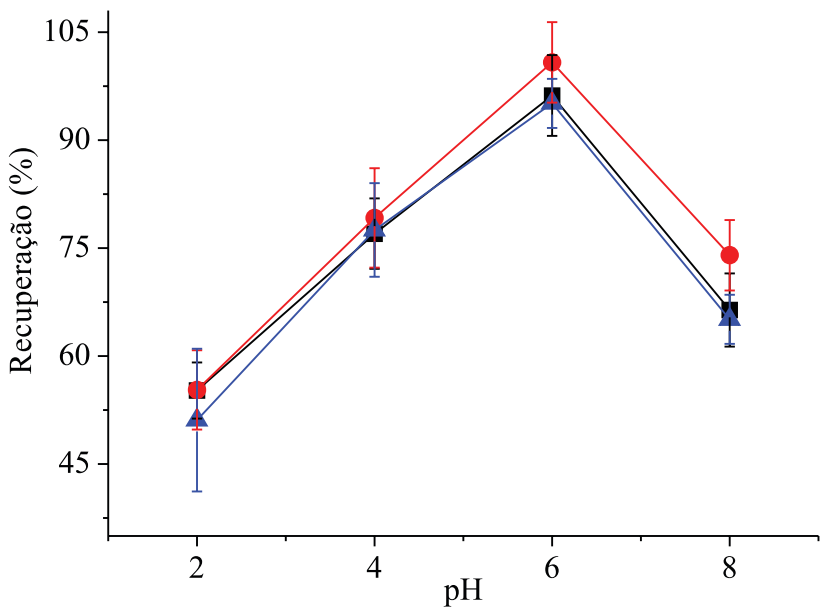

Figura 3. Recuperação dos agrotóxicos da fase Si(PDAS) em função do valor de $\mathrm{pH}$ da amostra de água. $n=3$. Carbendazim, $\boldsymbol{\bullet}$ diurom e $\boldsymbol{\Delta}$ parationa metílica

grandes de água podem lixiviar o composto inicialmente retido na fase sólida. O volume máximo de amostra de água para extração eficiente dos agrotóxicos, volume de breakthrough, foi avaliado para o método proposto. Volumes de amostra de 50 a $1000 \mathrm{~mL}$ foram estudados e os resultados apresentados na Figura 4.

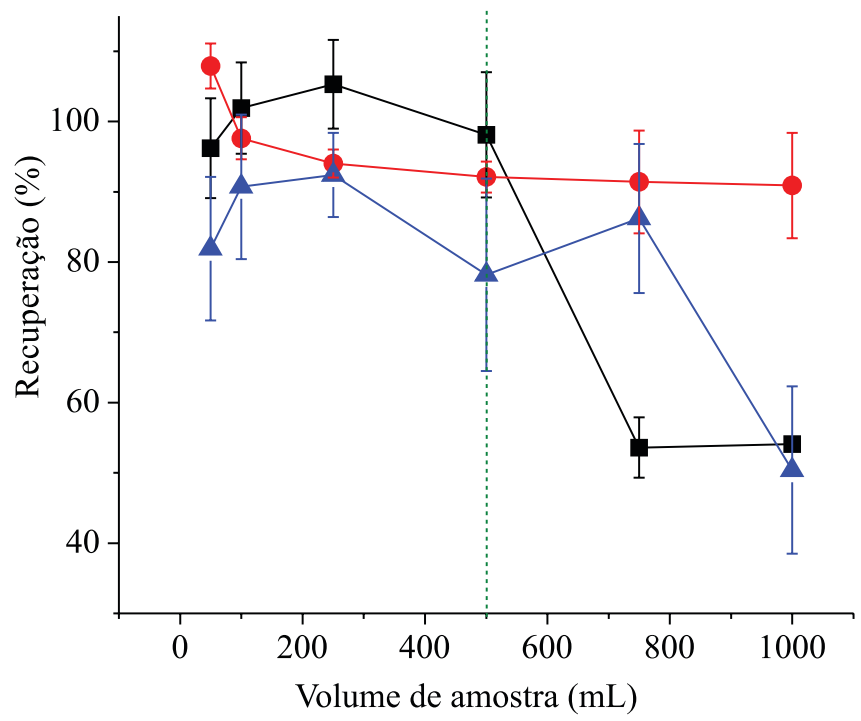

Figura 4. Volume de breakthrough para a recuperação dos agrotóxicos pela extração em fase sólida, empregando a fase Si(PDAS). $n=3 . \square$ Carbendazim,

- diurom e $\mathbf{\Delta}$ parationa metílica

O volume máximo de amostra a ser empregado no método foi definido como $500 \mathrm{~mL}$, uma vez que a partir desse volume a recuperação do carbendazim reduz significativamente para valores próximos de $50 \%$, ou seja, abaixo do mínimo da faixa de recuperação aceitável para resíduos de agrotóxicos de 70-120\%. ${ }^{17}$ Como o coeficiente de partição octanol-água do carbendazim é o menor dentre os agrotóxicos estudados, a passagem contínua de volumes muito grandes de água leva à lixiviação do composto pela maior solubilidade desse na água. A parationa metílica só começa a perder significativamente a sua taxa de recuperação a partir da eluição de $750 \mathrm{~mL}$ de amostra, enquanto que o diurom mantém a taxa constante mesmo após a passagem de $1000 \mathrm{~mL}$ de água.

O fator de concentração alcançado no método de extração dos agrotóxicos, portanto, foi de 1000 vezes, considerando os $500 \mathrm{~mL}$ de amostra de água eluídos pelos cartuchos $\mathrm{Si}(\mathrm{PDAS})$ e os $0,5 \mathrm{~mL}$ de metanol empregados na eluição dos agrotóxicos.

\section{Validação do método de determinação de agrotóxicos}

O método de extração em fase sólida, empregando os cartuchos lab-made $\mathrm{Si}(\mathrm{PDAS})$, de agrotóxicos de amostras de água otimizado consistiu na eluição de $500 \mathrm{~mL}$ de amostra de água ajustada para pH 6,00 e com $50 \mathrm{mmol} \mathrm{L}^{-1} \mathrm{de} \mathrm{Na}_{2} \mathrm{SO}_{4}$, empregando o hexano como solvente de limpeza e $0,5 \mathrm{~mL}$ de metanol para eluição dos compostos. O método otimizado foi submetido a um estudo de validação, avaliando os seguintes parâmetros analíticos: seletividade, linearidade, limites de detecção e quantificação, precisão (repetibilidade e reprodutibilidade em laboratório), exatidão (ensaios de recuperação) e robustez. O estudo de validação foi realizado de acordo com o protocolo experimental e critérios preconizados pelo documento SANTE/11945/2015. ${ }^{17}$

\section{Seletividade}

A seletividade do método foi determinada pela separação dos agrotóxicos no cromatograma, de modo que os picos referentes aos compostos não sofressem interferências entre si ou de componentes da matriz da amostra de água. A Figura 5 apresenta o cromatograma obtido na separação dos agrotóxicos estudados em uma amostra de água fortificada e submetida ao método de extração otimizada indicando que o método de análise foi seletivo para a determinação proposta.

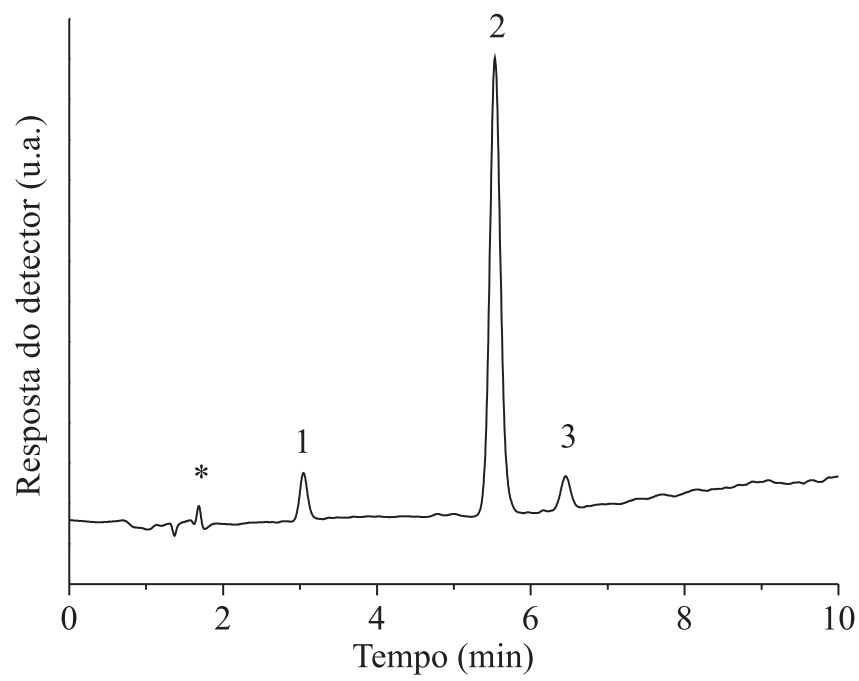

Figura 5. Cromatograma da separação de uma amostra de água fortificada com 0,5 $\mu \mathrm{g} \mathrm{mL}^{-1}$ de (1) carbendazim, (2) diurom e (3) parationa metílica e submetida ao método de extração SPE com Si(PDAS) otimizado. Condições cromatográficas: fase móvel MeOH: $\mathrm{H}_{2} \mathrm{O}$ com eluição por gradiente: gradiente: $0-3 \min 60 \% \mathrm{MeOH}$, 3-8 min, $90 \%$ de MeOHe 8-10 min 60\% MeOH. Vazão da fase móvel de 1,0 $\mathrm{mL} \mathrm{min}^{-1}$; temperatura de $25^{\circ} \mathrm{C}$; detecção UV a $237 \mathrm{~nm}$

\section{Faixa dinâmica linear, linearidade e sensibilidade}

Curvas analíticas para cada um dos agrotóxicos foram construídas por padronização externa, ou seja, fortificando amostras de água com diferentes concentrações dos compostos e submetendo-as ao método de determinação dos agrotóxicos proposta para o uso da fase sólida Si(PDAS). A partir das curvas analíticas, parâmetros como linearidade, sensibilidade e faixa dinâmica linear foram determinados e estão apresentados na Tabela 2.

O método proposto apresentou linearidade adequada das respostas obtidas para os agrotóxicos carbendazim, diurom e parationa metílica, com $r>0,99$, conforme critérios mais rigorosos estabelecidos pelas 
Tabela 2. Parâmetros obtidos das curvas analíticas dos agrotóxicos extraídos de amostras de água pelo método de extração em fase sólida com Si(PDAS) e análise por CLAE-UV

\begin{tabular}{lccc}
\hline Compostos & $\begin{array}{c}\text { Faixa dinâmica } \\
\text { linear }\left(\mu \mathrm{g} \mathrm{L}{ }^{-1}\right)\end{array}$ & $\begin{array}{c}\text { Coeficiente } \\
\text { linear }(\mathrm{r})\end{array}$ & $\begin{array}{c}\text { Sensibilidade } \\
\left(\mathrm{L} \mathrm{\mu g}^{-1}\right)\end{array}$ \\
\hline Carbendazim & $0,35-25,00$ & 0,999 & 0,211 \\
Diurom & $0,10-25,00$ & 0,995 & 1,561 \\
Parationa metílica & $0,40-25,00$ & 0,996 & 0,218 \\
\hline
\end{tabular}

agências reguladoras. ${ }^{20} \mathrm{O}$ método se mostrou mais sensível para o diurom quando comparado aos demais compostos. Estes valores de sensibilidade se refletem na faixa dinâmica linear para cada composto, em que se verifica a menor concentração quantificável (primeiro ponto da curva analítica) para o diurom frente aos demais compostos.

\section{Limites de detecção $(L D)$ e de quantificação $(L Q)$}

Os limites de detecção (LD) e de quantificação (LQ) do método de extração em fase sólida com o sorvente Si(PDAS) dos agrotóxicos de água foram determinados pelas equações.

$$
\mathrm{LD}=3,3 \times \mathrm{s} / \mathrm{S} \text { e } \mathrm{LQ}=10 \times \mathrm{s} / \mathrm{S}
$$

em que $s$ é o desvio padrão do coeficiente linear e $S$ o coeficiente angular da equação da curva analítica.

A partir das equações das curvas analíticas foram então obtidos os LD e LQ do método para cada um dos agrotóxicos estudados, Tabela 3. Os LQ foram confirmados a partir da injeção em triplicada de soluções padrão dos agrotóxicos preparadas nas concentrações equivalentes aos LQ do instrumento para cada composto, considerando o fator de concentração do método $\left(\mathrm{LQ}_{\text {método }} \mathrm{x} 1000\right)$.

Tabela 3. Limites de deteç̧ão e quantificação do método de extração em fase sólida com $\mathrm{Si}(\mathrm{PDAS})$ dos agrotóxicos de amostras de água, empregando a CLAE-UV. DPR é a estimativa do desvio padrão relativo do $\mathrm{LQ}_{\text {método }}$ para 3 medidas

\begin{tabular}{lccc}
\hline Compostos & $\mathrm{LD}_{\text {método }}\left(\mu \mathrm{g} \mathrm{L}^{-1}\right)$ & $\mathrm{LQ}_{\text {método }}\left(\mu \mathrm{g} \mathrm{L}^{-1}\right)$ & $\mathrm{DPR}(\%)$ \\
\hline Carbendazim & 0,10 & 0,35 & 7,5 \\
Diurom & 0,05 & 0,10 & 3,8 \\
Parationa metílica & 0,15 & 0,40 & 7,8 \\
\hline
\end{tabular}

Os valores de LQ do método foram determinados levando em consideração o fator de concentração da extração em fase sólida com o sorvente $\mathrm{Si}(\mathrm{PDAS})$ que foi de 1000 vezes. Assim, obtiveram-se $\mathrm{LQ}_{\text {met }}$ para o diurom como o menor entre os compostos estudados. Os valores de $\mathrm{LQ}_{\mathrm{met}}$ foram definidos de acordo com o recomendado pelo documento SANTE, ${ }^{17}$ em que a estimativa do desvio padrão relativo (DPR), para as injeções sucessivas na menor concentração quantificável para cada composto, ficou abaixo de $20 \%$. Os limites de detecção e quantificação obtidos para o método de extração em fase sólida empregando cartucho preparado no laboratório se mostraram competitivos em relação a outros métodos empregando sistemas de detecção mais sensíveis para agrotóxicos em água, devido principalmente ao alto fator de concentração alcançado com a SPE. ${ }^{21-23}$

\section{Precisão (repetibilidade e reprodutibilidade em laboratório) do método}

A precisão do método proposto foi avaliada segundo a sua repetibilidade e reprodutibilidade em laboratório, de acordo com o preconizado no documento SANTE/11945/2015. ${ }^{17}$ Para avaliar a repetibilidade do método, foram efetuadas extrações dos agrotóxicos fortificados nas amostras de água nas concentrações de 1, 3 e 5 vezes o $L_{\mathrm{met}}$ com cartuchos $\mathrm{Si}$ (PDAS). As extrações foram realizadas em 5 repetições, empregando o método proposto sob as mesmas condições experimentais. Na Tabela 4 são apresentadas as estimativas do desvio padrão relativo obtidas para cada agrotóxico, em três níveis de fortificação, após a extração em fase sólida com $\operatorname{Si}(\mathrm{PDAS})$ das amostras de água.

Tabela 4. Repetibilidade do método de extração em fase sólida de agrotóxicos de água, empregando CLAE-UV com cartuchos Si(PDAS) em diferentes níveis de fortificação. $\mathrm{n}=5$

\begin{tabular}{lccc}
\hline \multirow{2}{*}{ Compostos } & \multicolumn{3}{c}{ Repetibilidade (\% DPR) } \\
\cline { 2 - 4 } & $1 \times \mathrm{LQ}$ & $3 \times \mathrm{LOQ}$ & $5 \times \mathrm{LOQ}$ \\
\hline Carbendazim & 14 & 11 & 13 \\
Diurom & 10 & 5 & 5 \\
Parationa metílica & 8 & 6 & 2 \\
\hline
\end{tabular}

Os desvios padrão relativos obtidos nos ensaios de repetibilidade, Tabela 4, referem-se à dispersão dos resultados de recuperação de cada agrotóxico, para cinco repetições em cada nível de fortificação, empregando o método otimizado proposto neste trabalho. De acordo com o documento SANTE/11945/2015, ${ }^{17}$ um método de boa repetibilidade deve apresentar DPR inferior a $20 \%$ para o mínimo de cinco repetições, empregando as mesmas condições experimentais. Logo, o método de extração em fase sólida com o sorvente $\mathrm{Si}$ (PDAS) atende ao preconizado pelo documento, uma vez que a dispersão dos resultados, independente do composto, foi inferior a $14 \%$.

A reprodutibilidade em laboratório (ou precisão intermediária) do método proposto foi avaliada pela dispersão dos resultados (\% DPR) obtidos nas extrações dos agrotóxicos de água, para três níveis de fortificação, empregando o método otimizado no mesmo laboratório, pelo mesmo analista, nas mesmas condições de extração e análise, porém em três dias distintos. As estimativas dos desvios padrão relativos das recuperações obtidas nos três dias de estudo estão apresentadas na Tabela 5.

Tabela 5. Reprodutibilidade em laboratório do método de extração em fase sólida de agrotóxicos de água obtida em três dias distintos para diferentes níveis de fortificação. $\mathrm{n}=5$

\begin{tabular}{lccc}
\hline \multirow{2}{*}{ Compostos } & \multicolumn{3}{c}{ Reprodutibilidade em laboratório (\% DPR) } \\
\cline { 2 - 4 } & $1 \times$ LQ & $3 \times$ LOQ & $5 \times$ LOQ \\
\hline Carbendazim & 13 & 12 & 7 \\
Diurom & 10 & 4 & 3 \\
Parationa metílica & 10 & 16 & 10 \\
\hline
\end{tabular}

As estimativas dos desvios padrão relativos apresentados na Tabela 5 representam as dispersões das recuperações obtidas em três dias diferentes para cada nível de fortificação dos agrotóxicos estudados ao empregar o método otimizado neste trabalho. Os resultados indicam que não existe variação significativa da resposta, DPR $<17 \%$, quando o método é aplicado em dias distintos para extrair os agrotóxicos de amostras de água, estando em conformidade com o preconizado pelo documento SANTE, ${ }^{17}$ podendo, portanto, ser considerado como um método reprodutível dentro do laboratório.

\section{Exatidão do método (Ensaios de recuperação)}

Para avaliar a eficiência dos cartuchos Si(PDAS) em extrair e concentrar os resíduos de agrotóxicos de amostras de água pelo 
método proposto, amostras fortificadas em três níveis de concentração dos agrotóxicos: 1x, 3x e 5x LQ ${ }_{\text {met }}$ foram submetidas a ensaios de recuperação. A porcentagem de recuperação para cada agrotóxico foi determinada a partir da quantidade inicial adicionada dos padrões dos agrotóxicos nas amostras de água e a quantidade obtida nos extratos, de acordo com o grau de fortificação. De acordo com o documento SANTE/11945/2015, ${ }^{17}$ para que um método seja considerado de boa exatidão, a taxa de recuperação dos resíduos de agrotóxicos deve estar entre 70 a $120 \%$. A Tabela 6 apresenta a porcentagem de recuperação para cada agrotóxico a partir de 5 repetições em três diferentes níveis de fortificação da amostra após a aplicação do método proposto.

Tabela 6. Porcentagem de recuperação dos resíduos de agrotóxicos de amostras de água, empregando o método baseado na extração em fase sólida com cartuchos $\mathrm{Si}(\mathrm{PDAS}) . \mathrm{n}=5$

\begin{tabular}{lccc}
\hline \multirow{2}{*}{ Compostos } & \multicolumn{3}{c}{ Recuperação (\%) } \\
\cline { 2 - 4 } & $1 \times$ LQ & $3 \times$ LOQ & $5 \times$ LOQ \\
\hline Carbendazim & 76 & 89 & 96 \\
Diurom & 110 & 110 & 108 \\
Parationa metílica & 80 & 84 & 82 \\
\hline
\end{tabular}

Pelos valores obtidos de recuperação dos resíduos de agrotóxicos, Tabela 6, o método de extração dos agrotóxicos de água com os cartuchos Si(PDAS) apresenta boa exatidão. As porcentagens de recuperação, independente dos níveis de fortificação, ficaram na faixa de 76 a $110 \%$. Valores de recuperação acima de $100 \%$ são comumente obtidos por métodos de determinação de resíduos, uma vez que as concentrações medidas dos compostos são próximas do limite instrumental da técnica analítica, o que dificulta a integração do pico devido à variação do ruído instrumental.

\section{Extração em fase sólida de agrotóxicos de amostras de águas superficiais}

Após a otimização e a validação do método de determinação de resíduos de agrotóxicos de amostras de água, o método foi aplicado a amostras de água coletadas de três ribeirões na região do Pontal do Triângulo Mineiro, os quais cortam áreas de cultivo de cana-de-açúcar. As amostras de águas coletadas foram fortificadas com padrões dos agrotóxicos a 2,5 $\times \mathrm{LQ}_{\text {met }}$ e submetidas ao método otimizado e validado, empregando cartuchos $\mathrm{Si}(\mathrm{PDAS})$ preparados em laboratório e cartuchos $\mathrm{C}_{18}$ comerciais. Os resultados estão apresentados na Figura 6.

A recuperação dos resíduos de agrotóxicos de diferentes amostras de água coletadas em ribeirões do Triângulo Mineiro ficou sempre na faixa de 70-120\%, indicando que não há interferência da matriz da amostra de água no processo de determinação empregando o detector UV. Logo, o método analítico proposto não apresenta efeito matriz. Para fins de comparação da eficiência dos cartuchos Si(PDAS) preparados em laboratório, as amostras de água coletadas em ribeirões foram submetidas às mesmas condições de extração e análise, porém empregando cartuchos SPE comerciais tradicionais a base de $\mathrm{C}_{18}$. De acordo com a Figura 6 não houve variação significativa da resposta do método com a mudança do cartucho, confirmando a eficiência da extração dos cartuchos $\mathrm{Si}(\mathrm{PDAS})$, preparados em laboratório, porém com a vantagem de serem de fácil e simples preparação e, principalmente, de custo mais reduzido.

\section{CONCLUSÃO}

Neste trabalho buscou-se avaliar a aplicabilidade de cartuchos de extração em fase sólida preenchidos com o sorvente Si(PDAS),
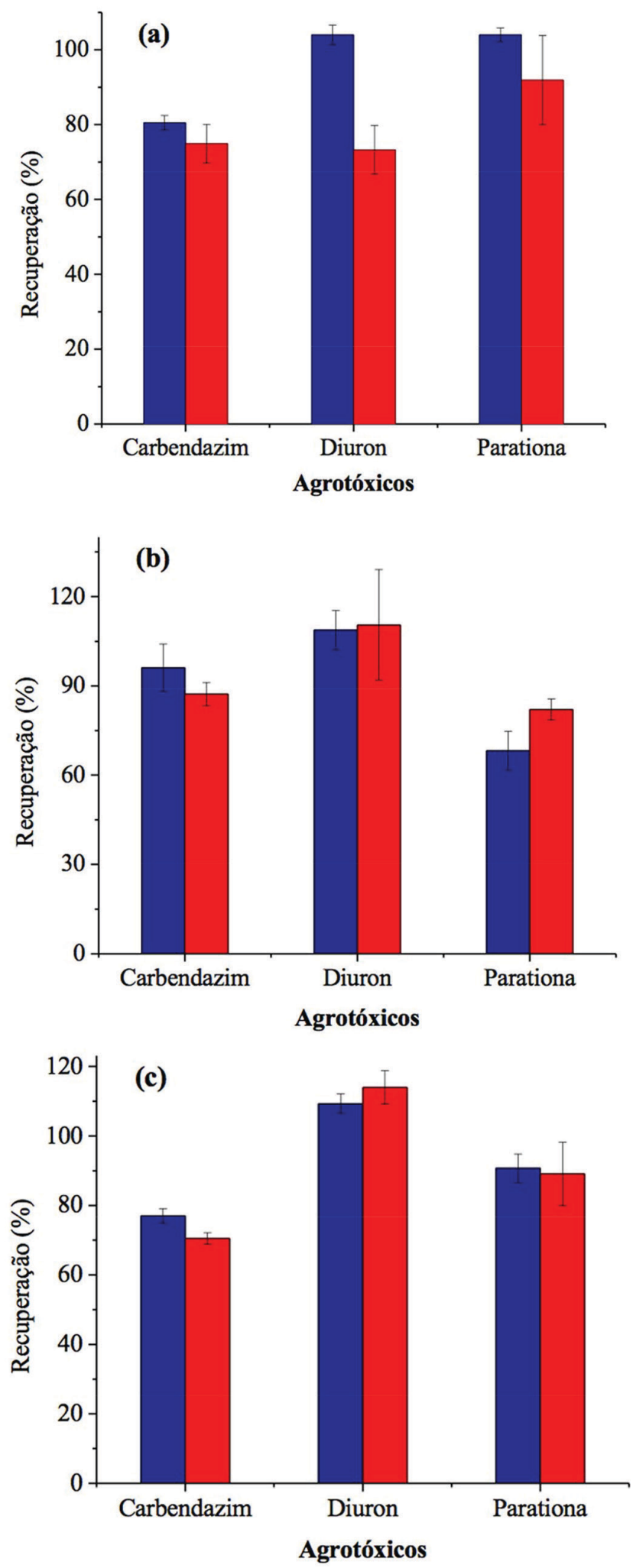

Figura 6. Recuperação dos resíduos de agrotóxicos das amostras (a) A, (b) $B$ e (c) C de água coletadas no Triângulo Mineiro, MG-Brasil, empregando o método de extração otimizado e validado com cartuchos $\square$ Si(PDAS) preparados em laboratório e $C_{18}$ comerciais

preparados em laboratório, em métodos de extração de agrotóxicos de amostras de água. O método foi otimizado, validado e aplicado a amostras de águas superficiais, mostrando que os cartuchos preparados em laboratórios são eficientes e comparáveis aos cartuchos 
comerciais tradicionais. Porém, a simplicidade e facilidade de preparação, bem como o baixo custo dos cartuchos $\mathrm{Si}(\mathrm{PDAS})$ preparados em laboratório, são os principais atrativos frente aos cartuchos convencionais. O método proposto empregando os cartuchos $\mathrm{Si}(\mathrm{PDAS})$ para a determinação de resíduos de agrotóxicos em amostras de águas superficiais se mostrou de boa exatidão e precisão e com níveis de detectabilidade comparáveis aos métodos que empregam técnicas analíticas sofisticadas, devido ao alto fator de concentração fornecido pela extração em fase sólida. O método pode ser considerado também robusto, uma vez que mais de 50 extrações foram realizadas ao longo de todo o estudo de validação, obtendo uma dispersão dos valores de recuperação inferior a $20 \%$ em termos de desvio padrão relativo.

\section{AGRADECIMENTOS}

À Fundação de Amparo à Pesquisa do Estado de Minas Gerais (FAPEMIG) pelo apoio financeiro.

\section{REFERÊNCIAS}

1. Toledo, M.; Lanças, F. M.; Carrilho, E.; J. Braz. Chem. Soc. 2007, 18, 1004.

2. Cavalcante, R. M.; Filho, N. S. M.; Viana, R. B.; Oliveira, I. R. N.; Nascimento, R. F.; Silveira, E. R.; Freire, G. S. S.; Quim. Nova 2007, 30,560 .

3. Jardim, I. C. S. F.; Sci. Chromatogr. 2010, 2, 13.

4. Liu, Q.; Shi, J.; Sun, J.; Wang, T.; Zeng, L.; Jiang, G.; Angew. Chem. 2011, 123, 6035 .

5. Naing, N. N.; Li, S. F. Y.; Lee, H. K.; J. Chromatogr. A 2016, 1427, 29.

6. Poiger, T.; Buerge, I. J.; Bächli, A.; Müller, M. D.; Balmmer, M. E.; Environ. Sci. Pollut. Res. 2017, 24, 1588.

7. Eiroa, A. A.; Canle, M.; Cancellieri, V. L.; Cerdà, V.; TrAC, Trends Anal. Chem. 2016, 80, 641.
8. Eiroa, A. A.; Canle, M.; Cancellieri, V. L.; Cerdà, V.; TrAC, Trends Anal. Chem. 2016, 80, 655.

9. Lindsey, M. E.; Meyer, M.; Thurman, E. M.; Anal. Chem. 2001, 73, 4640 .

10. Mozaz, S. R.; Alda, M. J. L; Barceló, D.; J. Chromatogr. A 2004, 1045, 85.

11. Kasprzyk-Hordern, B.; Dinsdale, R. M.; Guwy, A. J.; J. Chromatogr. A 2007, 1161, 132.

12. Dittmar, T.; Koch, B.; Hertkorn, N.; Kattner, G.; Limnol. Oceanogr.: Methods 2008, 6, 230.

13. Huo, S. H.; Yan, X. P.; Analyst 2012, 137, 3445.

14. Buszewski, B.; Szultka, M.; Crit. Rev. Anal. Chem. 2012, 42, 198.

15. Kirkland, J. J.; J. Chromatogr. A 2004, 1060, 9.

16. Novais, A. S.; Ribeiro Filho, J. F.; Amaral, E. M. F.; Faria, A. M.; Quim. Nova 2015, 38, 274.

17. European Commission. (2015). Document SANTE/11945/2015, Analytical quality control and method validation procedures for pesticide residues analysis in food and feed. Disponível em: http://www. eurl-pesticides.eu, acessada em Março 2018.

18. https://pubchem.ncbi.nlm.nih.gov/, acessada em Março 2018.

19. Silva, L. A.; Martins, C. R.; Andrade, J. B.; Quim. Nova 2004, 27, 1016.

20. Agência Nacional de Vigilância Sanitária - ANVISA; RE nº 899 de 29/05/2003: Guia para validação de métodos analíticos e bioanalíticos, Ministério da Saúde: Brasil 2003.

21. Raghu, P.; Reddy, T. M.; Swamy, B. E. K.; Chandrashekar, B. N.; Reddaiah, K.; Sreedhar, M.; J. Electroanal. Chem. 2012, 665, 76.

22. Ghanem, A.; Bados, P.; Perreau, F.; Benabdallah, R.; Plagellat, C.; Alencastro, L. F.; Einhorn, J.; Anal. Bioanal. Chem. 2008, 391, 345.

23. Wu, Q.; Li, Y.; Wang, C.; Liu, Z.; Zang, X.; Zhou, X.; Wang, Z.; Anal. Chim. Acta 2009, 638, 139. 